\title{
Sigmoid Sinus Characteristics Correlate with Early Clinical and Imaging Surrogates in Anterior Circulation Ischemic Stroke
}

\author{
Slaven Pikija ${ }^{1}$ - Jozef Magdic ${ }^{2}$ - David S. Liebeskind ${ }^{3}$ - Arthur Karamyan ${ }^{1}$. \\ Nele Bubel ${ }^{1}$ • Mark R. McCoy ${ }^{4}$ Johann Sellner ${ }^{1,5}$
}

Received: 14 July 2016 / Accepted: 30 August 2016/Published online: 10 September 2016

(C) The Author(s) 2016. This article is published with open access at Springerlink.com

\begin{abstract}
Cerebral venous outflow may play a decisive role in acute ischemic stroke. Here, we assessed the relation of cerebral sinus vein characteristics with clinical and imaging surrogates of early outcome in acute ischemic stroke. We evaluated cerebral vein characteristics in 212 patients with the middle cerebral artery (MCA) occlusive stroke confirmed by $\mathrm{CT}$ angiography CTA within $6 \mathrm{~h}$ from symptom onset. Readout parameters included volume and density of the sigmoid sinus (SS) and density of the superior sagittal sinus (SupSagS). These were correlated with early clinical outcome defined as hospital death (HD), final infarct volume (FIV), and National Institute of Health Stroke Scale (NIHSS) at discharge. We found a correlation for the volume of the right SS and the FIV when the M1 segment of the MCA of either side was occluded ( $p=0.002$, Rho $=0.206, n=134)$. A decrease in SS density was more pronounced in the subgroup with unfavorable outcome (NIHSS $>15+$ HD) but only when the left hemisphere was affected ( $p=0.026, n=101)$. On stepwise logistic regression analysis, adjusted for on-admission NIHSS, age at presentation, and FIV, smaller SS volume
\end{abstract}

Johann Sellner j.sellner@salk.at

1 Department of Neurology, Christian Doppler Medical Center, Paracelsus Medical University, Salzburg, Austria

2 Department of Neurology, Univerzitetni Klinični Center Maribor, Maribor, Slovenia

3 Neurovascular Imaging Research Core and UCLA Stroke Center, University of California, Los Angeles, CA, USA

4 Division of Neuroradiology, Christian Doppler Medical Center, Paracelsus Medical University Salzburg, Salzburg, Austria

5 Department of Neurology, Klinikum rechts der Isar, Technische Universität München, München, Germany was independently associated with lower odds for hospital death $(n=183$, OR $0.13,95 \%$ CI $0.02-0.94, p=0.043)$. A larger right SS and a decrease in density increase the risk of unfavorable early clinical and imaging outcome in AIS. This finding of an outflow pattern independent of the stroke site implicates an involvement of the cerebral venous drainage system in the pathophysiology of ischemic stroke.

Keywords Ischemic stroke $\cdot$ Cerebral veins $\cdot$ Biomarker . Densitometry $\cdot$ Outcome $\cdot$ Computed tomography

\section{Introduction}

Recent advances in vascular neuroimaging in patients with acute ischemic stroke (AIS) have led to the identification of prognostic factors. Determinants of outcome include the presence and density of a hyperdense artery sign, and location and extent of proximal artery occlusion [1,2]. There is also emerging evidence that good collateral circulation during AIS is associated with favorable clinical outcome, lesser extent of infarct volume, and success of revascularization measures [3-6]. The influence of venous structures and the basal venous sinuses, however, has been evaluated only scarcely in this context. Since the majority of the cerebral blood volume in the brain is contained in the venous pool, a restricted outflow may be of prognostic relevance. A recent study suggested that limitation of the ipsilateral venous drainage could contribute to development of edema in the setting of malignant middle cerebral artery (MCA) infarction [7]. Cortical venous drainage may also play a prognostic role for clinical outcome [8].

The great veins are interconnected and valveless, with the theoretical possibility of bidirectional blood flow. Blood from both cerebral hemispheres is drained mainly via the superior sagittal sinus into the confluence of sinuses and then via the 
transverse and sigmoid sinuses into the jugular bulb on the each side. A variable portion of the superficial temporal cortex is an exclusion and drains to the cavernous sinus and vein of Labbé to the ipsilateral distal or transversal sinus. However, this concept is challenged by significant anatomical variability and various extent of atresia [9, 10]. Whether the abnormalities of the venous system morphology are analogous to the consequences of an incomplete Willis circle has not been investigated so far [11]. The sigmoid sinus is known to be largely void of arachnoid granulations, thus allowing mostly unbiased analysis of its volume and density in simple axial plane on nonenhanced and enhanced CT images [12]. Here, we investigated a potential relationship of cerebral sinus vein characteristics with clinical and imaging surrogates of early outcome in acute ischemic stroke (AIS).

\section{Subjects and Methods}

We conducted this retrospective study at two Central European stroke centers. These were the Christian Doppler Medical Center in Salzburg, Austria (CDK) and the University Medical Centre in Maribor, Slovenia (MB). Only patients with $\mathrm{CT}$ angiography (CTA)-confirmed occlusion of M1 or M2 segment of the MCA were included. We excluded patients with CT scan performed more than $6 \mathrm{~h}$ after symptom onset, patients with unknown symptom onset time, and patients with additional pathologies such as brain tumors, arteriovenous malformations, and traumatic brain injury. Also excluded were patients with CT scans of low/unreadable quality. All data acquisition was done by purely retrospective chart review. Subsequent analyses were performed on anonymized data and therefore institutional review board approval was waived due to national regulations.

We recorded symptom-to-CT and time-to-control CT time points. NECT and CTA angiography scans were acquired at CDK with multidetector CT scanner Sensation 64 (Siemens, Erlangen, Germany) and in MB with multidetector CT scanner Aquilion 64 (Toshiba Medical Systems, Otawara, Japan). The NECT scans were reconstructed into 4-mm-thick adjacent slices through the whole brain. For CTA aquisition in MB and CDK $60 \mathrm{ml}$ Iomepron contrast agent was injected at a rate of $5 \mathrm{ml} / \mathrm{s}$ in $\mathrm{MB}$ and $4 \mathrm{ml} / \mathrm{s}$ in CDK and scanning would be triggered when contrast density in the arch of aorta was $180 \mathrm{HU}$ in MB and $100 \mathrm{HU}$ in CDK. Scanning time was set to $4-7 \mathrm{~s}$ in $\mathrm{MB}$ and $3 \mathrm{sec}$ in CDK. Axial NECT and CTA images were analyzed with the IMPAX tool. Evaluation of CT scans was performed at each site by experienced stroke physicians blinded to the clinical data.

\section{Institutional Standard Procedure with Acute Stroke Patients}

All patients underwent a stroke diagnostic pathway including brain CT diagnostics (NECT and CTA), blood examinations focusing on the identification of stroke etiology, transthoracal echocardiography, and 24-h ECG monitoring. Intravenous thrombolysis and mechanical thrombectomy were performed according to institutional guidelines.

\section{Quantification of the Superior Sagittal and Sigmoid Sinus Volume and Density}

The quantification of sinus volume and density is described in Fig. 1. Briefly, a round region of interest (ROI) was placed in the superior sagittal sinus (SupSagS) just above the lateral ventricles, and measurement of the average density in Hounsfield units were recorded (HU). We identified the right and left sigmoid sinuses (SS). The "freehand" ROI was placed inside the SS with the attention not to involve the bone and dural parts. Area and density measurements were then made on next two consecutive sections caudally on NECT and on the each second of the sections on CTA - due to thinner slices. Finally, the volume of the SS in cubic millimeters was determined by multiplying the measured areas with the distance between two slices. Validation experiments confirmed high interrater reliability of the method. Volumes of both SS were summed to calculate the summed SS volume (SUMSSV). Dominance of the sigmoid sinus was arbitrarily defined as the volume of the SS equal or larger than $80 \mathrm{~mm}^{3}$ from the opposite side.

\section{Derived Density of Sigmoid Sinuses Using CTA}

For CTA, we measured the density in the jugular bulb on both sides. The density of SupSagS was multiplied by 2 and then subtracted from sum of both SS densities in order to derive the calculated density of both SS (CDSS).

\section{SS Volume Ratio}

We calculated the SS volume ratio as follows: right SS volume/left SS volume. When the right SS was atretic, the ratio was given an arbitrary value of 0.01 . When the left SS was atretic, the ratio was given a value of 20 .

\section{Difference in Density Between SupSagS and Each SS-SSDelta}

In order to account for density differences between superior sagittal sinus and sigmoid sinus as a sign of possible outflow impairment, we calculated SSDelta value as SupSagS HU density - SS HU density separately for each side. However, SS could be atretic (nonexistent); in this case, SSDelta would 
Fig. 1 Schematic diagram of the cerebral venous system and the position of regions of interests (ROI) in nonenhanced brain CT (NECT) and CTA. In a first step, the superior sagittal sinus (SSS) average Hounsfield units (avg) values were recorded using the CT-A image. In the next step, a $\mathrm{ROI}$ is placed within the right sigmoid sinus on NECT. The bony part and the medial dural wall are avoided (black and white arrows). The A (area) in square millimeters and avg values are recorded. This procedure is repeated three times $(3 \times)$ to calculate sigmoid sinus volume in cubic millimeters and average density. $R$ right, $L$ left side

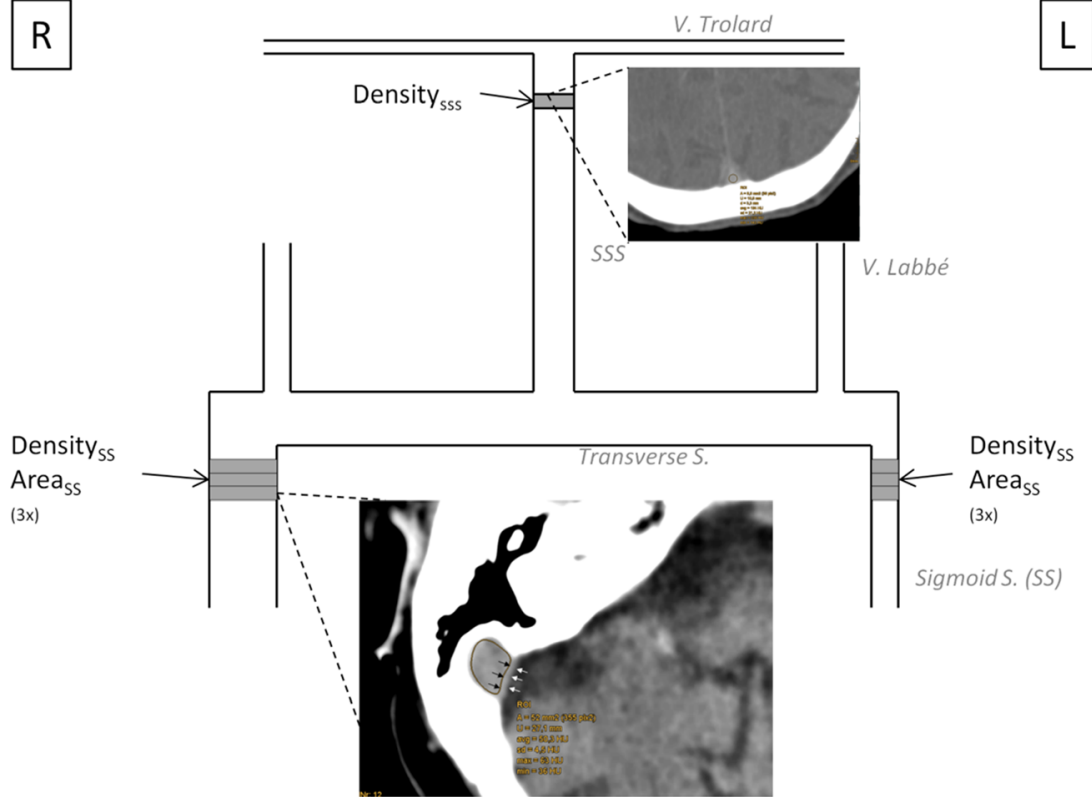

Quantification of Symptom Severity

be arbitrarily given the density value of SupSagS. With regard to the affected hemisphere, we considered two possibilities with regard to SSDelta value. The first option was when SSDelta on ipsilateral site was negative. This pattern was considered favorable since the ipsilateral sinus density was not lower than SupSagS. The second situation was when SSDelta on ipsilateral site was positive. This condition was considered unfavorable since the ipsilateral sinus density was not lower than SupSagS (Fig. 2).

\section{Quantification of the Final Infarct Volume}

The follow-up CT scans performed more than $24 \mathrm{~h}$ and less than 7 days were examined for presence of infarction, when present. The hypodense area was manually delineated on each CT slice (4-mm height) which produced the area in square centimeter. Finally, the volume in cubic centimeter was calculated from the measured area and the corresponding slice thickness.
The National Institutes of Health Stroke Scale (NIHSS) scoring on admission and transfer were available in the patient charts. Unfavorable outcome was defined as NIHSS $>15$ and hospital death (HD).

\section{Data Analysis}

Data about patients' demographics, medical history, imaging features, and clinical outcome were evaluated with descriptive statistics. Categorical variables were compared using Fisher's exact or $\chi^{2}$ test, while continuous variables were compared using the Mann-Whitney test for independent samples. The association between the variables was assessed by rank correlation. The relationship between different variables was analyzed by using multiple regression. In order to compare interrater HU and area measurement (reliabilities), we use intraclass correlation coefficient (ICC).

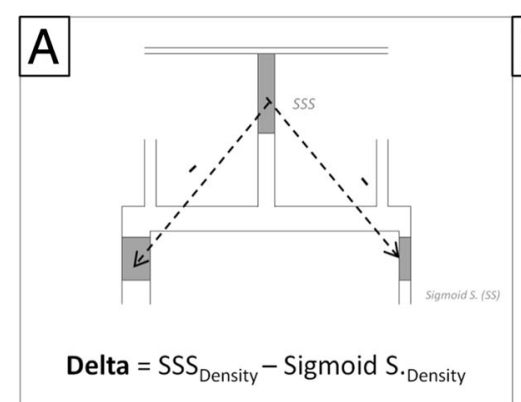

Fig. 2 Densitometry of sigmoid sinuses. Measurements of delta density in between SSS and sigmoid sinus (a). Delta negativity - in the setting of right middle cerebral artery stroke (MCA), there is no decrease of density

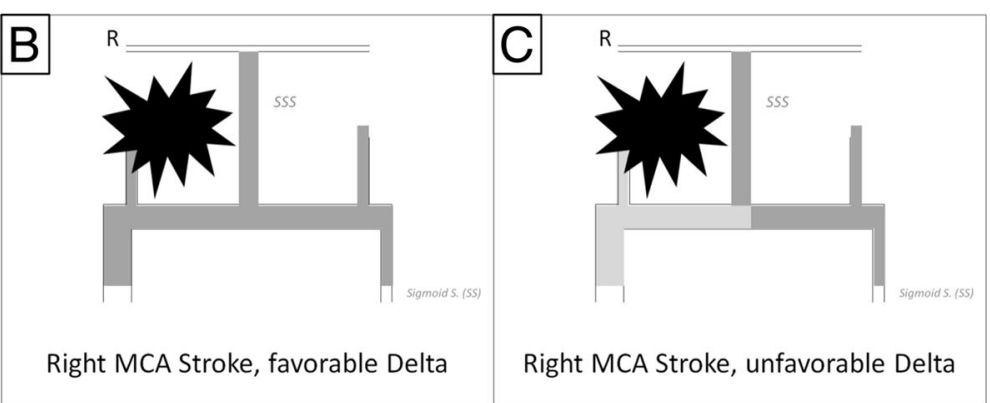

in right sigmoid sinus (favorable situation) (b). Unfavorable outcome, however, is associated with sigmoid sinus density decrease in respect to SSS density (c). SSS superior sagittal sinus 
All statistical analyses were performed using the STATA 13.0 software 2 (Science Plus Group, Groningen, The Netherlands).

\section{Results}

In Salzburg, we screened 2833 CT scans of consecutive patients with AIS admitted to CDK between June 2012 and December 2015 and identified 179 patients. In Maribor, 33 patients could be found by systematic search among 1796 consecutive patients with AIS in the period of January 2011 to January 2015. Among a total of 212 patients, CTA images were of sufficient quality in 197 cases $(92.9 \%)$. Patient demographics, treatment, and outcome are presented in Table 1.

The anatomical evaluation for different cerebral sinuses is shown in Table 2. SS volume (when measured) on the right and left sides showed a clear correlation between NECT and CTA measurements ( $p<0.001$ for both). The right SS was more often found to be present on CTA than on NECT ( $n=206,97.1 \%$ and $n=197,98.5 \%$, respectively). The left SS was detected on CT in $90.8 \%(N=206)$ and on CTA in $93.9 \%(N=197)$. The frequency of the atretic SS on CTA or NECT did not show side preferences in relation to the affected hemisphere.

\section{NECT}

The right SS was dominant in $52.4 \%$ and the left SS in $22.3 \%$. We noted codominance in $25.2 \%$. The final infarct volume did not differ between sex $(p=0.19)$ and had no correlation with the patients' age. Women had significant lower total SS volume than men (median 466.8 and $562.8 \mathrm{~cm}^{3}$, respectively; $p<0.001$ ).

\section{CTA}

The right SS was dominant in $50.8 \%$, the left SS in $25.9 \%$ and they were codominant in $23.3 \%$. We found a negative correlation of CDSS and SUMSSV, indicating that the larger sinuses had less contrast enhancement ( $p=0.002$, Rho $=-0.227$ ). Women had significantly lower SumSSV (median 692.0 vs. $566.8 \mathrm{~cm}^{3}, p<0.001$ ). The cases in the lowest quartile of SUMSSV (first $25 \%, n=50,25.4 \%$ ) tended to be younger. However, this observation did not reach statistical significance $(p=0.063)$.

\section{Interrater Agreement}

Interrater agreement for the NECT-measured venous densities in $\mathrm{HU}$ and areas of SS was determined in 20 randomly selected patients. The evaluation was performed by three raters for NECT (SP, AK, NB) and by two raters (SP, NB) for CTA. The
Table 1 Patient characteristics $(n=212)$

\begin{tabular}{|c|c|}
\hline Characteristics $(n)^{*}$ & Values \\
\hline \multicolumn{2}{|l|}{ Demographics (212) } \\
\hline Age (years) & $72(63,84)$ \\
\hline Women & $120(56.6)$ \\
\hline \multicolumn{2}{|l|}{ Medical history (212) } \\
\hline Prior stroke/TIA & $31(14)$ \\
\hline Atrial fibrillation & $107(50)$ \\
\hline Peripheral artery disease & $12(6)$ \\
\hline Carotid stenosis $>50 \%$ & $31(14)$ \\
\hline Arterial hypertension & $149(70)$ \\
\hline Diabetes mellitus & $32(15)$ \\
\hline Chronic heart failure & $40(19)$ \\
\hline Use of antiplatelets ${ }^{\dagger}$ & $57(27)$ \\
\hline Use of anticoagulants & $28(13)$ \\
\hline \multicolumn{2}{|l|}{ Stroke type by TOAST (212) } \\
\hline Cardioembolic & $115(54)$ \\
\hline Unknown & $52(25)$ \\
\hline Undetermined & $5(2)$ \\
\hline Other & $5(2)$ \\
\hline \multicolumn{2}{|l|}{ Clinical presentation } \\
\hline NIHSS (points) (212) & $16(11,20)$ \\
\hline Serum glucose (mmol/L) (210) & $118.8(106.0,141.0)$ \\
\hline Erythrocytes $\left(\times 10^{12} / \mathrm{L}\right)(179)$ & $4.5(4.1,4.8)$ \\
\hline HbA1c (mmol/L) (159) & $5.6(5.3,5.9)$ \\
\hline Fibrinogen (mg/dL) (208) & $355.5(306.0,436.0)$ \\
\hline C-reactive protein $(\mathrm{mg} / \mathrm{L})(159)$ & $0.4(0.18,1.0)$ \\
\hline \multicolumn{2}{|l|}{ Acute treatment (212) } \\
\hline Thrombolysis (rt-PA) & $156(74)$ \\
\hline Thrombectomy & $107(50)$ \\
\hline Thrombolysis + thrombectomy & $86(41)$ \\
\hline \multicolumn{2}{|l|}{ Thrombectomy outcome [TICI] (102) } \\
\hline No perfusion [0] & $18(18)$ \\
\hline Penetration, no distal filling [1] & $3(3)$ \\
\hline Perfusion, $<50 \%$ distal filling [2a] & $7(7)$ \\
\hline Inadequate $[0-2 \mathrm{a}$ total $]$ & $28(28)$ \\
\hline Perfusion, $>50 \%$ distal filling [2b] & $20(20)$ \\
\hline Full perfusion [3] & $54(53)$ \\
\hline Adequate $[2 \mathrm{~b}-3$ total $]$ & $64(63)$ \\
\hline \multicolumn{2}{|l|}{ Imaging particulars } \\
\hline \multicolumn{2}{|l|}{ Affected vessel (212) } \\
\hline Middle cerebral artery M1 & $154(73)$ \\
\hline Middle cerebral artery M2 & $58(27)$ \\
\hline Final infarct volume $\left(\mathrm{cm}^{3}\right)(197)$ & $41.2(9.9,134.1)$ \\
\hline \multicolumn{2}{|l|}{ Control image finding (201) } \\
\hline Infarction & $154(77)$ \\
\hline Hemorrhagic infarction & $33(16)$ \\
\hline Resolution (infarct volume $=0$ ) & $14(7)$ \\
\hline In-hospital mortality (212) & $40(19)$ \\
\hline NIHSS at discharge (points) (172) & $7.5(4,15)$ \\
\hline
\end{tabular}

Data are presented as median $(25,75$ percentile) or count (percent)

HbAlc glycated hemoglobin, NIHSS National Institutes of Health Stroke Scale, $r t-P A$ recombinant human tissue plasminogen activator, TIA transitory ischemic attack, TICI thrombolysis in cerebral infarction grading, TOAST Trial of Org 10172 in Acute Stroke Treatment 
Table 2 Measurement of cerebral venous sinuses with nonenhanced CT and CT angiography $(n=212)$

\begin{tabular}{|c|c|c|c|c|c|}
\hline \multirow{2}{*}{$\begin{array}{l}\text { Modality } \\
\text { Sinus/vein }\end{array}$} & \multicolumn{2}{|l|}{ CT } & \multirow{2}{*}{$\begin{array}{l}\text { Modality } \\
\text { Sinus/vein }\end{array}$} & \multicolumn{2}{|l|}{ CT angiography } \\
\hline & Volume $\left(\mathrm{mm}^{3}\right)$ & Density in HU & & Volume $\left(\mathrm{mm}^{3}\right)$ & Density in HU \\
\hline Sigmoid right (200) & $305.0(191.4,392.8)$ & $50.4(47.0,55.2)$ & Sigmoid right (194) & $357.4(239.6,470.4)$ & $131.8(99.7,171.8)$ \\
\hline Sigmoid left (187) & $212.4(136.0,299.2)$ & $51.1(47.0,56.2)$ & Sigmoid left (185) & $277.2(160.0,375.6)$ & $141.7(104.5,176.0)$ \\
\hline Sagittal sinus (210) & - & $56.6(51.4,60.5)$ & Sagittal sinus (199) & - & $176.0(132.0,215.0)$ \\
\hline- & - & - & Jugular bulb right (188) & - & $102.0(77.5,141.0)$ \\
\hline- & - & - & Jugular bulb left (172) & - & $110.0(74.8,143.0)$ \\
\hline
\end{tabular}

Data are presented as median $(25,75$ percentile) or count (percent)

$H U$ Hounsfield units

agreement for SupSagS density as measured on NECT was excellent: $\mathrm{ICC}=0.88$, for density and area of right $\mathrm{SS}$ $\mathrm{ICC}=0.92$ and $\mathrm{ICC}=0.85$, respectively, and for density and area of left SS ICC $=0.98$ and $\mathrm{ICC}=0.79$, respectively. On CTA, the SupSagS density correlation was good (ICC $=0.77$ ). The ICC for the density of the right SS and area of the right $\mathrm{SS}$ were $\mathrm{ICC}=0.90$ and $\mathrm{ICC}=0.77$, respectively. The density and area of the left SS ICCs were ICC $=0.95$ and $\mathrm{ICC}=0.95$, respectively.

\section{Volumetric Measurements}

\section{CTA and Clinical Outcome}

Patients in the lowest quartile of combined SS volume $(n=50)$ when compared with others had less HD (6.0 and $22.4 \%$, $p=0.009)$ and less unfavorable outcome (22.0 vs. $43.5 \%$, $p=0.007)$. When the left SS was dominant, the risk of hospital death was higher when the ipsilateral (left) hemisphere was affected ( $n=101, p=0.017$ ). The side of atresia (or volume below $5 \%$ ) in respect to the affected hemisphere had no relation to clinical outcome.

\section{CTA and Imaging Outcome}

We identified a correlation for right SS volume and the FIV when the M1 segment of either side was occluded ( $p=0.002$, Rho $=0.206, n=134)$. This association was even more pronounced when the left MCA M1 was occluded ( $p=0.036$, Rho $=0.259, n=66)$ and weaker for the right-sided M1 occlusion ( $p=0.159, n=68$ ). The left SS volume did not show a correlation with FIV. We detected an impact of the left SS volume on FIV when the ratios of the SS volumes were analyzed. The ratio of the right SS volume divided by the left SS volume (rSS volume/lSS volume) showed correlation with FIV $(n=183$, $p=0.036$ ), as shown in Fig. 3 .

\section{CT and Imaging Outcome}

The volume of the right SS was positively correlated with the FIV when MCA M1 of either side was occluded ( $p=0.046$, Rho $=0.169, n=139$ ). This positive association remained present when right MCA M1 was occluded at $(p=0.028$, Rho $=0.259, n=72$ ). Yet, no such correlation was found for the left MCA M1 occlusion ( $p=0.159, n=68)$. No association for the left SS volume and FIV was found.

\section{Densitometric Measurements}

\section{CTA and Clinical Outcome}

We found a trend for the correlation of the density of the right SS and unfavorable outcome in the ipsilateral MCA M1 occlusion $(p=0.051)$. There was also a trend for SSDelta negativity (the ipsilateral SS sinus was more dense than SupSag) and favorable outcome $(p=0.055)$.

\section{CTA and Imaging Outcome}

The density of the right SS correlated with FIV in the setting of the ipsilateral M1 occlusion $(p=0.036)$. The left SS volume did not show a correlation with FIV.

\section{Multivariate Analysis}

The smaller volume of both SS (SUMSSV) as measured on CTA was significantly associated with less odds for hospital death $(n=183$, OR $0.13,95 \%$ CI $0.02-0.94, p=0.043)$ on stepwise logistic regression analysis, including on-admission NIHSS, age at presentation, and FIV. When FIV was excluded from the analysis, the SS volume was associated with smaller odds for unfavorable clinical outcome $(n=197$, OR 0.32 , $95 \%$ CI $0.12-0.85, p=0.023$ ). 
Fig. 3 Correlation of right sinus sigmoideus (SS) volume, expressed as ratio between the right and left SS volume, with final infarct volume in 183 acute stroke patients, $p=0.036$

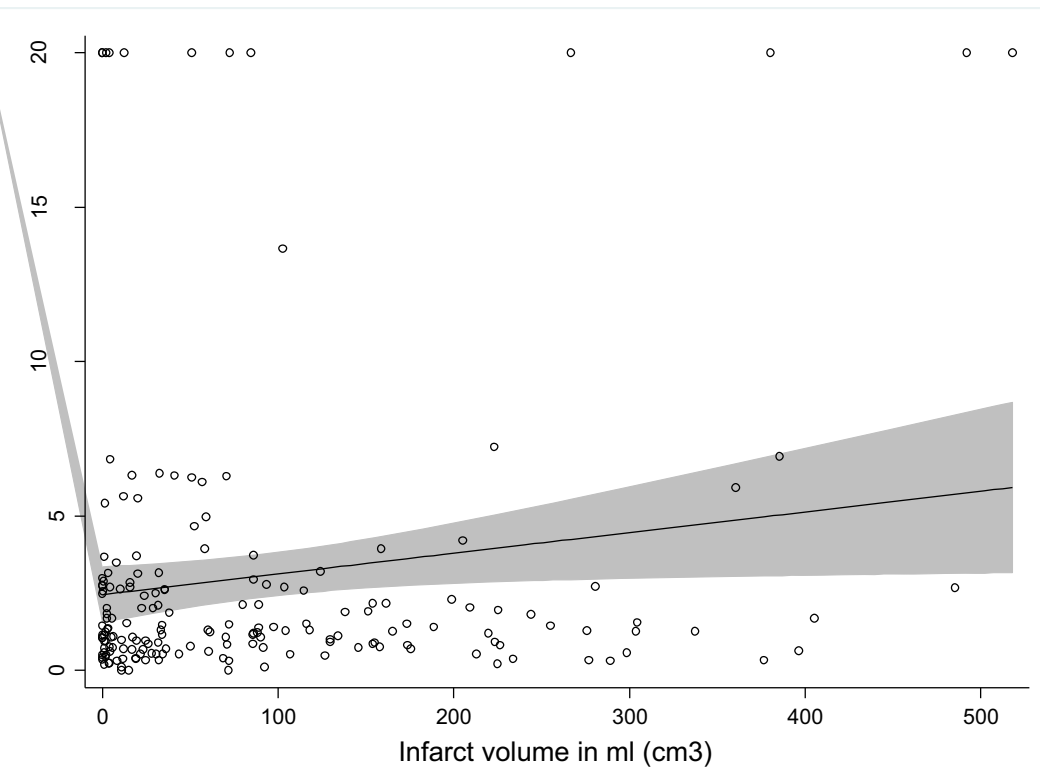

\section{Discussion}

These novel findings establish the potential role and impact of the structural status of the cerebral venous system in anterior circulation AIS. The volumetric analysis of the SS revealed univariate and multivariate associations with early clinical and imaging outcomes. In this regard, our study disclosed that a larger right SS in terms of pronounced asymmetry is associated with hospital death and the larger final infarct volume in the setting of MCA M1 occlusion of either side. Densitometry on the other hand showed weaker associations with the outcome surrogates. In the univariate analysis, the decrease in density of the ipsilateral sigmoid sinus to the MCA infarct showed a trend for unfavorable outcome. In the univariate analysis, the right SS density showed a negative association with the FIV. Thus, we propose a potential role of venous outflow in the pathophysiology of ischemic stroke.

The findings of this study extend the previous work that showed significance of impaired cortical venous outflow as measured on the CTA on prognosis in acute stroke [8]. It was established that density, especially those of the superficial intracerebral veins, predicts late outcome (90 day) even when adjusted for baseline NIHSS score. In our sample, the density of the right SS correlated with FIV when the ipsilateral hemisphere was affected.

It is known that atresia of one of the sinuses could lead to impaired venous outflow, thus worsening brain edema and leading to unfavorable outcome [7]. However, reports in this direction are only available on small number of patients and results were positive only for atresia/thrombosis ipsilateral to affected hemisphere. In our series, we could not confirm these findings for early clinical or imaging outcomes and pure or near-atresia (defined as the volume of SS under $5 \%$ of the group).
As proposed by Liebeskind et al., the elevated pressure in venous system could provide heightened downstream resistance for therapeutic flow augmentation [13]. This is corroborated indirectly by our study showing that lower density of SS translates to worse outcome. We can consider ipsilateral density as a surrogate marker for heightened venous pressure. Notably, in our study, only 4/21 (19\%) cases without decrease in SS density had unfavorable clinical outcome.

Dural sinuses and deep cerebral veins are capable to reverse flow in physiologic situations mainly in elderly population [14]. This possibility could provide compensatory outflow resistance via retrograde flow thus offsetting the collapse of the cerebral veins and potentially eliminating blood flow maldistribution [15]. There is a possibility as suggested by our work that the disproportionality between SSs could impair such possible collateralization.

The proposed measurements of the SS characteristics are fast and practical and could be done within $5 \mathrm{~min}$. Moreover, it is highly reproducible as shown by interrater correlation data. The limitations of the current study are few but important. The sample is limited only to the cases with performed CTA, thus biasing the population to the more affected individuals where this examination was judged as necessary. However, the study was performed in two university centers, thus reducing additional selection bias. Moreover, we did not account for other accessory venous outflow patterns such as cervical epidural venous system through anterior condylar confluent system. However, this is a rare constellation [16, 17]. Furthermore, we used CTA technique that is not time-resolved, and as the consequence, the measured density of the venous compartment decreases from sagittal sinus to jugular bulb. In this regard, we needed to account for atresic sinuses. Regardless, we sought to determine the density of the sigmoid sinus in 
order to extrapolate the amount of venous outflow. Eventually, future studies should evaluate the outcome beyond the acute phase, e.g., after 3 months.

In conclusion, we have extended the previous observation of impact of impaired superficial venous outflow on clinical outcome. The outflow pattern we identified for unfavorable prognosis was independent from the site of stroke and, thus, may play a decisive role in the early pathophysiology of stroke. Further studies, especially time-resolved ones reflecting directionality of the venous outflow in the setting of arterial pathology are mandatory to shed more light on this neglected but emerging theme.

Acknowledgments Open access funding provided by Paracelsus Medical University.

\section{Compliance with Ethical Standards}

Conflict of interest The authors declare that they have no competing interests.

\section{References}

1. Qazi E, Al-Ajlan FS, Najm M, Menon BK (2016) The role of vascular imaging in the initial assessment of patients with acute ischemic stroke. Curr Neurol Neurosci Rep 16(4):32. doi:10.1007 /s11910-016-0632-y

2. Tan CC, Wang HF, Ji JL, Tan MS, Tan L, Yu JT (2016) Endovascular treatment versus intravenous thrombolysis for acute ischemic stroke: a quantitative review and meta-analysis of 21 randomized trials. Mol Neurobiol. doi:10.1007/s12035-016-9738-0

3. Nambiar V, Sohn SI, Almekhlafi MA, Chang HW, Mishra S, Qazi E, Eesa M, Demchuk AM, et al. (2014) CTA collateral status and response to recanalization in patients with acute ischemic stroke. AJNR Am J Neuroradiol 35(5):884-890. doi:10.3174/ajnr.A3817

4. Leng X, Fang H, Leung TW, Mao C, Xu Y, Miao Z, Liu L, Wong $\mathrm{KS}$, et al. (2016) Impact of collateral status on successful revascularization in endovascular treatment: a systematic review and metaanalysis. Cerebrovasc Dis 41(1-2):27-34. doi:10.1159/000441803

5. Yang CY, Chen YF, Lee CW, Huang A, Shen Y, Wei C, Liu HM (2008) Multiphase CT angiography versus single-phase CT angiography: comparison of image quality and radiation dose. AJNR Am J Neuroradiol 29(7):1288-1295. doi:10.3174/ajnr.A1073
6. Chen H, Liu N, Li Y, Wu B, Su Z, Zhu G, Hu J (2016) The role of dynamic contrast-enhanced MR in evaluating level III collateral circulation in a rat model of acute ischemic stroke. Mol Neurobiol. doi:10.1007/s12035-016-9844-z

7. Yu W, Rives J, Welch B, White J, Stehel E, Samson D (2009) Hypoplasia or occlusion of the ipsilateral cranial venous drainage is associated with early fatal edema of middle cerebral artery infarction. Stroke J Cereb Circ 40(12):3736-3739. doi:10.1161 /STROKEAHA.109.563080

8. Parthasarathy R, Kate M, Rempel JL, Liebeskind DS, Jeerakathil T, Butcher KS, Shuaib A (2013) Prognostic evaluation based on cortical vein score difference in stroke. Stroke J Cereb Circ 44(10): 2748-2754. doi:10.1161/STROKEAHA.113.001231

9. Doepp F, Schreiber SJ, von Munster T, Rademacher J, Klingebiel R, Valdueza JM (2004) How does the blood leave the brain? A systematic ultrasound analysis of cerebral venous drainage patterns. Neuroradiology 46(7):565-570. doi:10.1007/s00234-004-1213-3

10. Beards SC, Yule S, Kassner A, Jackson A (1998) Anatomical variation of cerebral venous drainage: the theoretical effect on jugular bulb blood samples. Anaesthesia 53(7):627-633

11. van Seeters T, Hendrikse J, Biessels GJ, Velthuis BK, Mali WP, Kappelle LJ, van der Graaf Y, Group SS (2015) Completeness of the circle of Willis and risk of ischemic stroke in patients without cerebrovascular disease. Neuroradiology 57(12):1247-1251. doi:10.1007/s00234-015-1589-2

12. Roche J, Warner D (1996) Arachnoid granulations in the transverse and sigmoid sinuses: CT, MR, and MR angiographic appearance of a normal anatomic variation. AJNR Am J Neuroradiol 17(4):677-683

13. Liebeskind DS (2009) Venous hemodynamics may enhance collateral perfusion and the fibrinolytic milieu in paradoxical embolism. Stroke J Cereb Circ 40(2):e30-e31. doi:10.1161 /STROKEAHA.108.541441

14. Uchino A, Nomiyama K, Takase Y, Nakazono T, Tominaga Y, Imaizumi T, Kudo S (2007) Retrograde flow in the dural sinuses detected by three-dimensional time-of-flight MR angiography. Neuroradiology 49(3):211-215. doi:10.1007/s00234-006-0186-9

15. Pranevicius M, Pranevicius O (2002) Cerebral venous steal: blood flow diversion with increased tissue pressure. Neurosurgery 51(5): 1267-1273 discussion 1273-1264

16. Stoquart-Elsankari S, Lehmann P, Villette A, Czosnyka M, Meyer ME, Deramond H, Baledent O (2009) A phasecontrast MRI study of physiologic cerebral venous flow. J Cereb Blood Flow Metab 29(6):1208-1215. doi:10.1038/sj. jcbfm.jcbfm200929

17. San Millan Ruiz D, Gailloud P, Rufenacht DA, Delavelle J, Henry F, Fasel JH (2002) The craniocervical venous system in relation to cerebral venous drainage. AJNR Am J Neuroradiol 23(9):1500 1508 